\title{
Worsened survival in the head-up tilt position cardiopulmonary resuscitation in a porcine cardiac arrest model
}

\author{
Yong Joo Park', Ki Jeong Hong ${ }^{2}$, Sang Do Shin², Tae Yun Kim³, \\ Young Sun Ro ${ }^{4}$, Kyoung Jun Song ${ }^{2}$, Hyun Ho Ryu ${ }^{5}$ \\ 'Department of Emergency Medicine, Gyeongsang National University Changwon Hospital, Gyeongsang \\ National University School of Medicine, Changwon, Korea \\ ${ }^{2}$ Department of Emergency Medicine, Seoul National University Hospital, Seoul, Korea \\ ${ }^{3}$ Department of Emergency Medicine, Gyeongsang National University School of Medicine, Jinju, Korea \\ ${ }^{4}$ Laboratory of Emergency Medical Services, Seoul National University Hospital Biomedical Research \\ Institute, Seoul, Korea \\ ${ }^{5}$ Department of Emergency Medicine, Chonnam National University School of Medicine, Gwangju, Korea
}

Objective Head elevation at an angle of $30^{\circ}$ during cardiopulmonary resuscitation (CPR) was hemodynamically beneficial compared to supine position in a previous porcine cardiac arrest experimental study. However, survival benefit of head-up elevation during CPR has not been clarified. This study aimed to assess the effect of head-up tilt position during CPR on 24-hour survival in a porcine cardiac arrest experimental model.

Methods This was a randomized experimental trial using female farm pigs ( $n=18,42 \pm 3 \mathrm{~kg}$ ) sedated, intubated, and paralyzed on a tilting surgical table. After surgical preparation, 15 minutes of untreated ventricular fibrillation was induced. Then, 6 minutes of basic life support was performed in a position randomly assigned to either head-up tilt at $30^{\circ}$ or supine with a mechanical CPR device, LUCAS-2, and an impedance threshold device, followed by 20 minutes of advanced cardiac life support in the same position. Primary outcome was 24-hour survival, analyzed by Fisher exact test.

Results In the 8 pigs from the head-up tilt position group, one showed return of spontaneous circulation (ROSC); all eight pigs expired within 24 hours. In the eight pigs from the supine position group, six had the ROSC; six pigs survived for 24 hours and two expired. The head-up position group showed lower 24-hour survival rate and lower ROSC rate than supine position group $(\mathrm{P}<0.01)$.

Conclusion The use of head-up tilt position with 30 degrees during CPR showed lower 24-hour survival than the supine position.

Keywords Heart arrest; Cardiopulmonary resuscitation; Animal experimentation
elSSN: 2383-4625

Received: 18 July 2018

Revised: 9 September 2018

Accepted: 16 September 2018

Correspondence to: Ki Jeong Hong Department of Emergency Medicine, Seoul National University Hospital, 101 Daehak-ro, Jongno-gu, Seoul 03080, Korea

E-mail: ssberg@snu.ac.kr ORCID

http://orcid.org/0000-0003-3334-817X

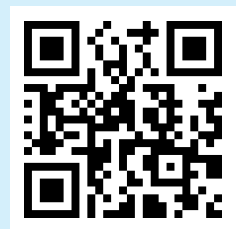

How to cite this article: Park YJ, Hong KJ, Shin SD, Kim TY, Ro YS, Song KJ, Ryu HH. Worsened survival in the head-up tilt positional cardiopulmonary resuscitation in porcine cardiac arrest model. Clin Exp Emerg Med 2019;6(3):250-256.
This is an Open Access article distributed under the terms of the Creative Commons Attribution Non-Commercial License (http:// creativecommons.org/licenses/by-nc/4.0/). 


Capsule
Summary
$\begin{aligned} & \text { What is already known } \\ & \text { In previous study, head elevation during cardiopulmonary resuscitation (CPR) decreased intracranial pressure and im- } \\ & \text { proved cerebral perfusion pressure. However, clinical outcome including return of spontaneous circulation (ROSC) rate } \\ & \text { and survival has not been elucidated. }\end{aligned}$
$\begin{aligned} & \text { What is new in the current study } \\ & \text { In our investigation, we assessed the effect of head-up elevation during CPR on 24-hour survival and ROSC rate. CPR in } \\ & \text { the head-up elevated position at an angle of } 30^{\circ} \text { showed lower 24-hour survival rate and ROSC rate than that in supine } \\ & \text { position. }\end{aligned}$

\section{INTRODUCTION}

Out of hospital cardiac arrest occurs frequently in residential areas in Asian countries, and many victims are required to be transported from high-rise buildings in metropolitan cities. ${ }^{1-4}$ During transport from high-rise buildings, the position of the cardiac arrest victim could be head-up position in elevators or stairs. Mechanical compression devices can be used to perform cardiopulmonary resuscitation (CPR) during prehospital transport. Transport from high-rise buildings requires longer time from the scene of cardiac arrest to the ambulance vehicle ${ }^{5}$; Additionally, the lowflow or no-flow time may get lengthened in such situations.

A previous study reported that head-up tilt position at an angle of $30^{\circ}$ during CPR had hemodynamic benefits with respect to coronary perfusion pressure (CoPP) and cerebral perfusion pressure (CePP), as compared to supine or head-down position. ${ }^{6}$ The hemodynamic benefits of higher CoPP is associated with a higher rate of return of spontaneous circulation (ROSC). ${ }^{7.8}$ Higher CePP during CPR is shown to have increased the survival rate. ${ }^{9}$ However, the association between head-up tilt position during CPR and 24-hour survival is still unknown.

The aim of this study was to assess the association between head-up tilt position with a $30^{\circ}$ angle and 24-hour survival using a porcine cardiac arrest experimental model. The results of our study can be used to predict the association of clinical outcome and transport of cardiac arrest victims in the head-up tilt position for long durations from high rise buildings.

\section{METHODS}

\section{Study design}

This was a large animal experimental study using the porcine cardiac arrest model. It was approved by the Institutional Animal Care and Use Committee of the institution where the study was performed (approval no. 14-0195-S1A0). All animal care complied with the Laboratory Animal Act of the Korean Ministry of Food and Drug Safety. A certified and licensed veterinarian assured that the experiment was performed within the approved protocol and guidelines.

\section{Surgical preparation}

Female Yorkshire farm pigs weighing $42 \pm 3 \mathrm{~kg}$, were selected. They were observed for at least 1 week at the research facility and were fasted overnight prior to experiment. Pigs were initially sedated with intramuscular injection of Zoletil $(2 \mathrm{mg} / \mathrm{kg}$ ) and xylazine $(2 \mathrm{mg} / \mathrm{kg})$ at the neck, followed by inhaled isoflurane (5\%) via fitting mask. Pigs were positioned on a table with their legs tied to the ends of the tilt table. The tilt table was specially built to tilt a pig's whole body longitudinally, and to be locked at a designated degree with a bolted-in backboard to connect to the mechanical CPR device to support ongoing CPR during the position change.

Endotracheal intubation was performed using a $7 \mathrm{~mm}$ internal diameter endotracheal tube. Isoflurane at $1 \%$ to $2 \%$ was administered continuously via endotracheal tube during the overall surgical preparation period. A burr-hole was drilled in the skull between the occipital prominence and the right eyebrow to place a Mikro-tip (Millar, Houston, TX, USA.) pressure catheter in the subdural space, to monitor and measure intracranial pressure (ICP). A Mikro-tip pressure catheter was inserted through the right or left femoral artery to mid-cardiac level of thoracic aorta to measure the aortic blood pressure. Another Mikro-tip pressure catheter was placed through the external jugular vein in the right atrium to record the right atrial pressure. Tip position was confirmed by pressure tracings.

Pigs were ventilated with a volume-control ventilator adjusted at a tidal volume of $12 \mathrm{~mL} / \mathrm{kg}$ and a respiratory rate of 12 times/ $\mathrm{min}$, and were continuously monitored and appropriately adjust- 
ed to maintain a $\mathrm{PaCO}_{2}$ above $40 \mathrm{mmHg}$ and $\mathrm{PaO}_{2}$ above $80 \mathrm{mmHg}$. An intravenous heparin bolus (100 units/kg) and an infusion of normal saline $(1,000 \mathrm{~mL})$ were also given. The central temperature monitored by rectal temperature was maintained between $37.5^{\circ} \mathrm{C}$ to $38.0^{\circ} \mathrm{C}$ using a temperature-control blanket (Blanketrol II; Cincinnati Sub-Zero Medical Division, Cincinnati, OH, USA). Electrocardiography, oxygen saturation monitoring from ear lobe surface, and partial pressure of end-tidal $\mathrm{CO}_{2}$ levels were monitored and recorded throughout the experiment.

All data measurements were recorded using a digital data acquisition system (PowerLab; AD Instruments, Colorado Springs, CO, USA).

\section{Experiment protocol}

Ventricular fibrillation (VF) was induced by introducing direct current using a pacing wire to the right ventricle through the exter- nal jugular vein. When VF was initiated, the ventilator was disconnected immediately, and the pig was untreated for 15 minutes in the supine position (Fig. 1). During this time, a computergenerated randomization of supine or head- up $30^{\circ}$ position was assigned.

After the untreated VF period of 15 minutes, the position was randomly assigned to supine or head-up tilt position with $30^{\circ}$ (Fig. 2). Then, basic life support (BLS) was performed for 6 minutes using the LUCAS 2 Chest Compression System (Jolife $A B$, Lund, Sweden) device at a compression rate of 100 times/minute and compression depth of 4 to $5 \mathrm{~cm}$. Respiratory rate at 10 breaths per minute was delivered by resuscitator bagging. An impedance threshold device with a resistance of $10 \mathrm{~cm} \mathrm{H}$ O (ResQPOD ITD; Advanced Circulatory Systems, Roseville, MN, USA) was attached to the endotracheal tube during the overall resuscitation period.

After 6 minutes of CPR, the pig was defibrillated with 200 joule

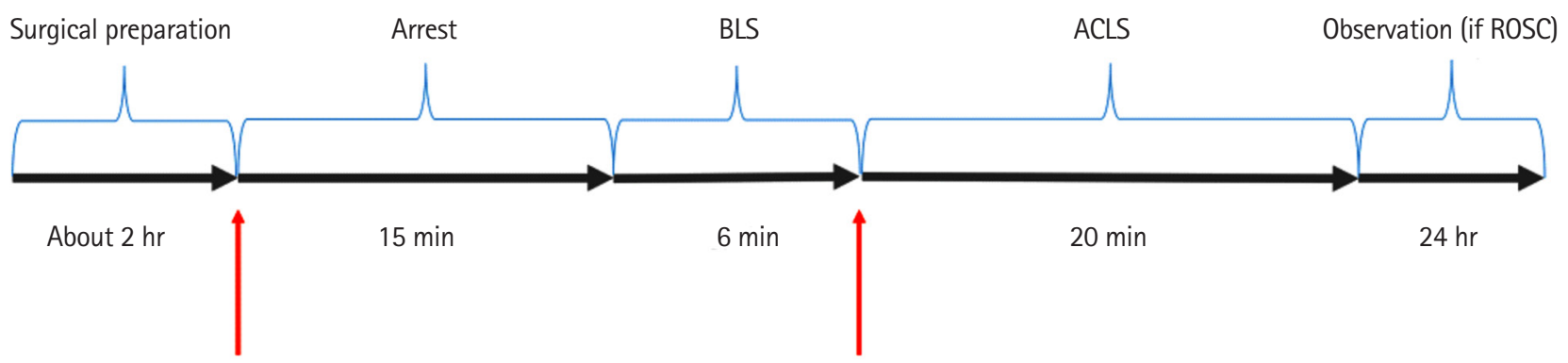

Randomization

First attempt of

VF induction electrical defibrillation

Fig. 1. Experimental protocol. BLS, basic life support; ACLS, advanced cardiac life support; ROSC, return of spontaneous circulation; VF, ventricular fibrillation.

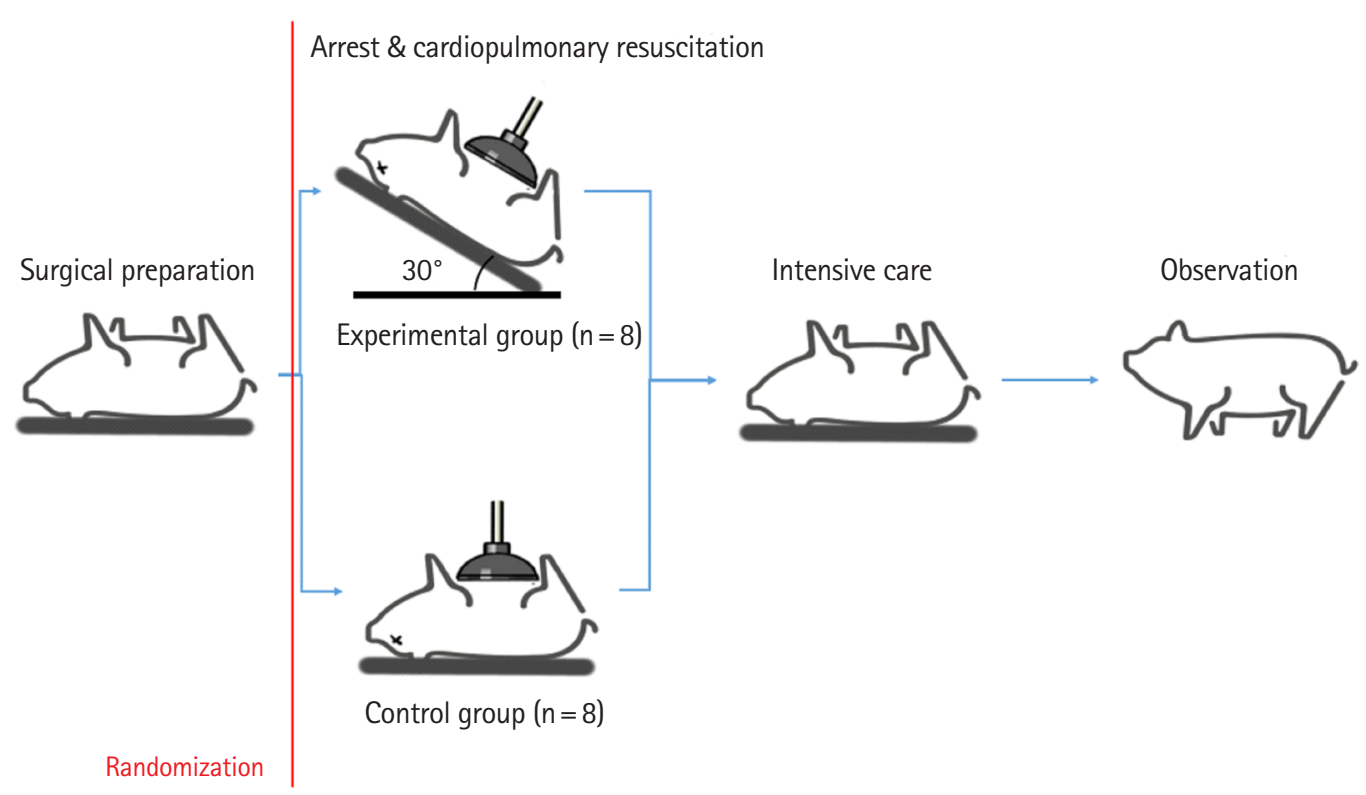

Fig. 2. Brief scheme of the experiment. 
biphasic shocks, if a shockable rhythm was observed. If there was no ROSC, additional CPR was performed for up to 20 minutes. During additional CPR, defibrillation was performed every 2 minutes, and epinephrine was injected with every 3 minutes. If there was no ROSC within the additional 20-minute CPR period, the pig was pronounced dead, and an autopsy was performed. If there was ROSC, the pig was resuscitated intensively by hydration and additional epinephrine for up to 60 to 90 minutes until it showed spontaneous self-breathing. When the respiration rate was maintained above 10 times/min, it was extubated and transferred to the cage, and observed for 24 hours. Upon completion of the experiment protocol, pigs were then sacrificed with a $20-\mathrm{mL}$ injection of saturated potassium chloride. If severe intrathoracic injury including hemothorax or severe lung contusion was noted in autopsy result, we excluded the animal from the final analysis.

\section{Outcome measurements}

We collected hemodynamic profiles continuously throughout the trial. CePP was calculated as the difference between mean aortic pressure (MAP) and mean ICP. CoPP was calculated as the difference between right atrial pressure and aortic pressure during the CPR decompression phase. Base-line hemodynamic data were measured within 1 minute before induction of VF. During 6 minutes of BLS, hemodynamic data were measured every 1 minute.

The primary outcome was 24-hour survival. The secondary outcome was ROSC rate after BLS for 6 minutes. Two trained experimental researchers determined 24-hour survival and ROSC.

\section{Statistical analysis}

The sample size was estimated by assuming a 60\% survival in the experimental group and 10\% survival in the control group with alpha-error of 0.05 , and statistical power of $0.8 .^{8}$ The estimated sample size was 17 pigs in each group, with 34 pigs overall.

A reassessment of the survival rate was scheduled after every 6th experiment. If an extreme difference in the survival rate between experimental group and control group was noted in the reassessment, the experiment would be terminated before reaching the estimated sample size, to reduce unnecessary animal sacrifices.

Fisher exact test was performed to determine the survival difference between supine and head-up tilt position groups. A Pvalue less than 0.05 was considered statistically significant.

\section{RESULTS}

We initially included 18 pigs in the study. Two pigs, no. 9 and no. 16 , were excluded because of hemothorax and severe lung contusion, respectively. After the 18th experiment, an extreme difference in the survival rate between experimental group and control group was observed and the study was terminated as planned. Sixteen pigs were finally included for analysis.

Basic characteristics and hemodynamic parameters of each positional group are described in Table 1. There was no significant difference between the two groups. There was also no difference in overall elapsed time for surgical procedures between the two groups (Table 2).

Hemodynamics of both groups during the 6 minutes of BLS period are described in Tables 3 and 4 . In the head-up tilt position

Table 1. Baseline characteristics and hemodynamics in each position group

\begin{tabular}{lccc}
\hline Parameter & $\begin{array}{c}\text { Head-up tilt } \\
\text { position }(n=8)\end{array}$ & $\begin{array}{c}\text { Supine position } \\
(n=8)\end{array}$ & P-value \\
\hline Body weight $(\mathrm{kg})$ & $44.1 \pm 3.3$ & $43.2 \pm 3.7$ & 0.61 \\
Rectal temperature $\left({ }^{\circ} \mathrm{C}\right)$ & $36.9 \pm 1.2$ & $36.7 \pm 0.9$ & 0.71 \\
Heart rate $(/ \mathrm{min})$ & $99.5 \pm 21.3$ & $100.5 \pm 12.8$ & 0.91 \\
$\mathrm{SpO}_{2}(\%)$ & $98.0 \pm 1.2$ & $97.7 \pm 1.2$ & 0.62 \\
$\mathrm{ETCO}_{2}(\mathrm{mmHg})$ & $42.0 \pm 4.1$ & $39.8 \pm 6.8$ & 0.44 \\
Respiration rate $(/ \mathrm{min})$ & $14.3 \pm 2.7$ & $14.0 \pm 1.6$ & 0.85 \\
Mean ICP $(\mathrm{mmHg})$ & $14.0 \pm 2.6$ & $15.0 \pm 4.4$ & 0.58 \\
MAP $(\mathrm{mmHg})$ & $80.9 \pm 9.9$ & $85.2 \pm 13.2$ & 0.47 \\
Systolic RAP $(\mathrm{mmHg})$ & $6.0 \pm 1.6$ & $6.1 \pm 1.2$ & 0.88 \\
\hline
\end{tabular}

$\mathrm{SpO}_{2}$, percutaneous $\mathrm{O}_{2}$ saturation; $\mathrm{ETCO}_{2}$, end-tidal $\mathrm{CO}_{2} ; \mathrm{ICP}$, intracranial pressure; MAP, mean arterial pressure; RAP, right atrial pressure.

Table 2. Elapsed time for surgical procedures in each position group

\begin{tabular}{|c|c|c|c|}
\hline Parameter & Head-up tilt position $(n=8)$ & Supine position $(n=8)$ & P-value \\
\hline Elapsed time for ear vein cannulation (min) & $1.5 \pm 2.5$ & $1.6 \pm 3.1$ & 0.94 \\
\hline Elapsed time for intubation (min) & $5.0 \pm 4.8$ & $3.3 \pm 2.3$ & 0.38 \\
\hline Elapsed time for ICP monitoring catheter insertion (min) & $19.9 \pm 6.6$ & $20.5 \pm 9.5$ & 0.88 \\
\hline Elapsed time for AoP monitoring catheter insertion (min) & $34.1 \pm 33.4$ & $20.0 \pm 7.3$ & 0.26 \\
\hline Elapsed time for RAP monitoring catheter insertion (min) & $28.1 \pm 13.1$ & $23.3 \pm 8.8$ & 0.40 \\
\hline Elapsed time for VF induction (min) & $2.2 \pm 1.7$ & $4.1 \pm 5.0$ & 0.32 \\
\hline Total elapsed time before VF induction (min) & $176.3 \pm 43.4$ & $160.5 \pm 35.1$ & 0.43 \\
\hline
\end{tabular}

$I C P$, intracranial pressure; AoP, aortic pressure; $R A P$, right atrial pressure; $V F$, ventricular fibrillation. 
Table 3. Hemodynamics during basic life support and outcomes of supine position group

\begin{tabular}{lcccccccc}
\hline Order of experiment & MAP $(\mathrm{mmHg})$ & $\mathrm{ICP}(\mathrm{mmHg})$ & $\mathrm{dAoP}(\mathrm{mmHg})$ & $\mathrm{dRAP}(\mathrm{mmHg})$ & CePP $(\mathrm{mmHg})$ & CoPP $(\mathrm{mmHg})$ & ROSC & 24-hour survival \\
\hline 1 & 42.4 & 21.4 & 26.5 & -2.1 & 20.9 & 28.7 & + & Survived \\
4 & 29.2 & 14.8 & 9.8 & 7.0 & 14.3 & 2.9 & - & Expired \\
6 & 37.7 & 27.3 & 26.1 & 10.1 & 10.5 & 16.0 & + & Survived \\
7 & 29.3 & 17.7 & 18.7 & 7.5 & 11.6 & 11.2 & - & Expired \\
10 & 36.3 & 16.1 & 26.7 & 19.4 & 20.2 & 7.3 & + & Survived \\
13 & 33.4 & 17.9 & 27.1 & 5.6 & 15.5 & 21.6 & + & Survived \\
15 & 40.1 & 21.0 & 34.0 & 5.3 & 19.1 & 25.4 & + & Survived \\
17 & 47.1 & 21.8 & 40.7 & 6.2 & 25.2 & 34.5 & + & Survived \\
\hline
\end{tabular}

MAP, mean arterial pressure; ICP, intracranial pressure; dAoP, diastolic phase aortic pressure; dRAP, diastolic phase right atrial pressure; CePP, cerebral perfusion pressure; CoPP, coronary perfusion pressure; ROSC, return of spontaneous circulation.

Table 4. Hemodynamics during basic life support and outcomes of head-up tilt position group

\begin{tabular}{lcccccccc}
\hline Order of experiment & MAP $(\mathrm{mmHg})$ & $\mathrm{ICP}(\mathrm{mmHg})$ & $\mathrm{dAoP}(\mathrm{mmHg})$ & $\mathrm{dRAP}(\mathrm{mmHg})$ & CePP $(\mathrm{mmHg})$ & CoPP $(\mathrm{mmHg})$ & ROSC & 24-hour survival \\
\hline 2 & 31.0 & -3.9 & 7.9 & -3.6 & 34.9 & 11.5 & - \\
3 & 19.9 & -6.6 & 4.6 & -8.7 & 26.5 & 13.3 & - \\
5 & 11.1 & -6.9 & 7.1 & -3.4 & 18.1 & 10.5 & - \\
8 & 20.0 & -5.5 & 8.8 & -4.5 & 25.5 & 13.3 & - \\
11 & 4.6 & -7.6 & -5.8 & -2.7 & 12.3 & -3.1 & - & Expired \\
12 & 20.3 & -0.5 & 14.2 & -10.0 & 20.8 & 24.1 & Expired \\
14 & 17.4 & 0.06 & 5.1 & -6.6 & 17.3 & 11.7 & - & - \\
18 & 20.1 & -8.2 & 1.3 & -2.2 & 28.4 & 3.5 & Expired & Expired \\
\hline
\end{tabular}

$M A P$, mean arterial pressure; ICP, intracranial pressure; dAoP, diastolic phase aortic pressure; dRAP, diastolic phase right atrial pressure; CePP, cerebral perfusion pressure; $\mathrm{CoPP}$, coronary perfusion pressure; ROSC, return of spontaneous circulation.

Table 5. Comparison of hemodynamics and clinical outcomes between the 2 position groups

\begin{tabular}{lccr}
\hline & $\begin{array}{c}\text { Head-up tilt } \\
\text { position }(\mathrm{n}=8)\end{array}$ & $\begin{array}{c}\text { Supine position } \\
(\mathrm{n}=8)\end{array}$ & P-value \\
\hline Hemodynamic profiles & & & \\
MAP $(\mathrm{mmHg})$ & $18.0 \pm 7.6$ & $36.9 \pm 6.2$ & $<0.01$ \\
ICP $(\mathrm{mmHg})$ & $-4.8 \pm 3.1$ & $19.7 \pm 3.9$ & $<0.01$ \\
dAoP $(\mathrm{mmHg})$ & $5.4 \pm 5.9$ & $26.2 \pm 9.2$ & $<0.01$ \\
dRAP $(\mathrm{mmHg})$ & $-5.2 \pm 2.9$ & $7.8 \pm 5.9$ & $<0.01$ \\
CePP $(\mathrm{mmHg})$ & $22.9 \pm 7.2$ & $17.1 \pm 5.0$ & 0.08 \\
CoPP $(\mathrm{mmHg})$ & $10.6 \pm 7.9$ & $18.4 \pm 11.0$ & 0.12 \\
Clinical outcome & & & \\
ROSC & 1 & 6 & 0.04 \\
24-hour survival & 0 & 6 & $<0.01$ \\
\hline
\end{tabular}

MAP, mean arterial pressure; ICP, intracranial pressure; $d A o P$, diastolic phase aortic pressure; dRAP, diastolic phase right atrial pressure; CePP, cerebral perfusion pressure; CoPP, coronary perfusion pressure; ROSC, return of spontaneous circulation.

group $(n=8)$, one pig had ROSC, but expired after 24-hours (Fig. 3). All pigs expired in the head-up tilt position group. In the supine position group $(n=8)$, six pigs had ROSC and all of them survived for 24-hours.

MAP, ICP, diastolic phase aortic pressure (dAoP) and diastolic phase right atrial pressure (dRAP) were higher in the supine group
(Table 5). CePP was higher in the head-up group but this was not significant. The head-up tilt position group had a lower 24-hour survival rate and lower ROSC rate than the supine position group $(\mathrm{P}<0.05)$.

\section{DISCUSSION}

This study used a porcine experimental cardiac arrest model to compare the ROSC rate and 24-hour survival rate between headup tilt position and supine positions. In our study, the head-up tilt positioned CPR group showed lower ROSC rate and lower 24hour survival rate. In terms of hemodynamic profile, the head-up tilt positioned group had lower MAP, ICP, dAoP, and dRAP. This suggests that head-up tilt positioned CPR during transport of cardiac arrest victims in elevator or stairs in high-rise buildings could worsen hemodynamic profiles and clinical outcome during CPR.

The $30^{\circ}$ head-up tilt group showed higher CePP than the supine group but this was not significant. The $30^{\circ}$ head-up tilt group showed lower MAP, ICP, dAoP, and dRAP. Such poorer hemodynamics could worsen the clinical outcome in the head-up tilt positioned CPR group. A previous study assessed the benefit of the head-up tilt position on increased CePP with lower ICP. However, 

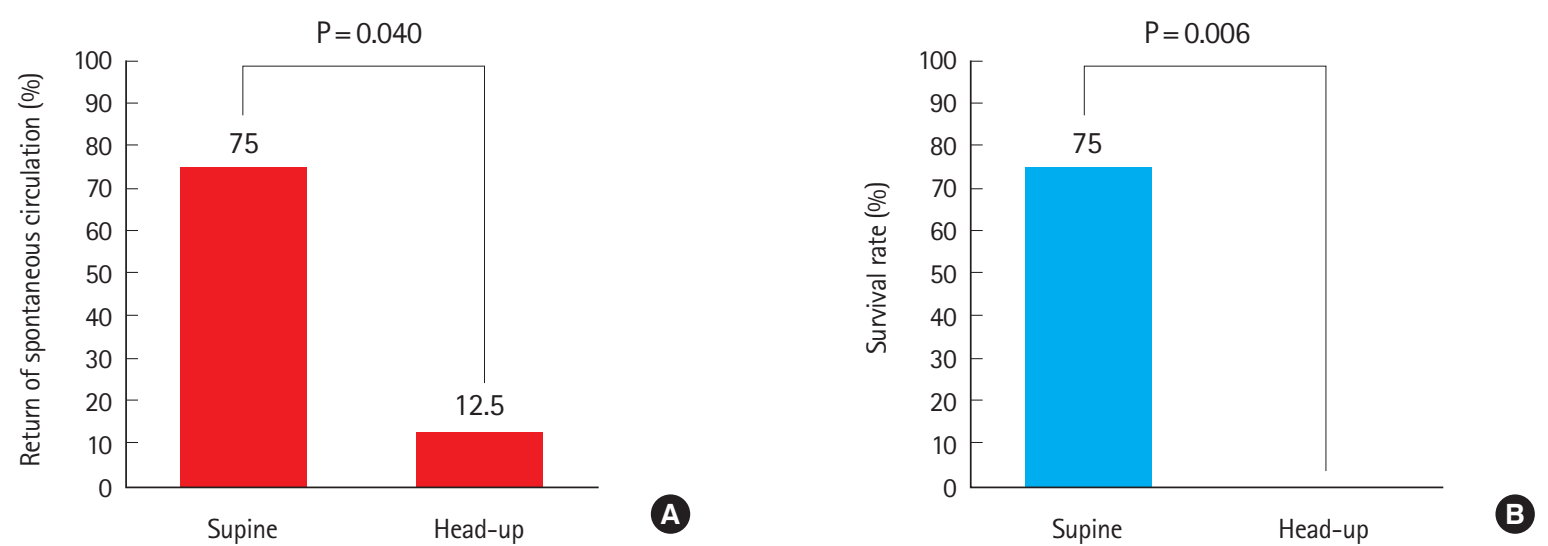

Fig. 3. Clinical outcomes between the two position groups. (A) Return of spontaneous circulation rate of each group and (B) survival rate of each group.

in our experiment, the benefit of lower ICP was not significantly associated with increased CePP. In contrast, low MAP, dAoP, and dRAP was observed in the head-up tilt position indicating poor venous return during CPR in the head-up tilt position.

In our study protocol, untreated VF time was 15 minutes. Longer untreated VF time could affect hemodynamic profiles and clinical outcome. In cases where cardiac arrest occurred in highrise buildings, the response time increased and the rate of occurrence of low-flow or no-flow time increased..$^{9}$ Thus, a relatively long duration of CPR with the head-up position could occur in prehospital response to cardiac arrest in high-rise buildings. Our study implies that poorer hemodynamics for long durations with no-flow time in the head-up tilt position could result in poor clinical outcome of cardiac arrest victims located in high-rise buildings.

The head-up tilt position with a $30^{\circ}$ angle was achieved with whole body elevation of the pig. A recent study reported that head and neck elevation of the pig resulted in higher CePP and CoPP than in the supine positioned group. ${ }^{10,11}$ This suggests that the whole body tilted head-up position used in this protocol could worsen hemodynamics more than the elevated position limited to the head and neck.

There was also the possibility of anatomical considerations causing poor outcome in the head-up tilt group. When cardiac arrest occurs, skeletal muscles such as the diaphragm are paralyzed, and gravity could easily pull down intra-thoracic organs such as the heart and lung. This suggests that the chest compression point could have changed when head-up tilt CPR was performed. This also could affect intra-thoracic pressure during cardiac arrest. In the porcine cardiac arrest model, passive leg raising was not associated with hemodynamic benefits. Tourniquet assisted CPR may overcome poor venous return during head-up CPR. ${ }^{12}$ More investigation is required on this.
This study had several limitations. First, this is an animal experimental trial and had the limitation of generalizability in humans. Second, the anatomy of the pig is different from the human body. To set up the head-up tilt position for pigs, the upper limbs were severely stretched by ropes to hang the body on the tilted table. These experimental methods did not comply with the normal physiology of the pig. Third, we assessed the sample size to assess the association of survival between the two groups. However, our study terminated before completion of the planned sample size of the experiments.

In conclusion, head-up tilt positioned CPR with a $30^{\circ}$ angle showed lower 24-hour survival rate and lower ROSC rate than supine positioned CPR in our porcine cardiac arrest experimental model.

\section{CONFLICT OF INTEREST}

No potential conflict of interest relevant to this article was reported.

\section{REFERENCES}

1. McNally $B$, Robb $R$, Mehta $M$, et al. Out-of-hospital cardiac arrest surveillance: Cardiac Arrest Registry to Enhance Survival (CARES), United States, October 1, 2005 - December 31, 2010. MMWR Surveill Summ 2011;60:1-19.

2. Lateef $F$, Anantharaman $V$. Delays in the EMS response to and the evacuation of patients in high-rise buildings in Singapore. Prehosp Emerg Care 2000;4:327-32.

3. Morrison $\sqcup$, Angelini MP, Vermeulen MJ, Schwartz B. Measuring the EMS patient access time interval and the impact of responding to high-rise buildings. Prehosp Emerg Care 2005; 9:14-8. 
4. Silverman RA, Galea $S$, Blaney $S$, et al. The "vertical response time": barriers to ambulance response in an urban area. Acad Emerg Med 2007;14:772-8.

5. Conway $A B, M c D a v i d ~ A$, Emert JM, et al. Impact of building height and volume on cardiac arrest response time. Prehosp Emerg Care 2016;20:212-9.

6. Debaty G, Shin SD, Metzger A, et al. Tilting for perfusion: headup position during cardiopulmonary resuscitation improves brain flow in a porcine model of cardiac arrest. Resuscitation 2015;87:38-43.

7. Reynolds JC, Salcido DD, Menegazzi JJ. Coronary perfusion pressure and return of spontaneous circulation after prolonged cardiac arrest. Prehosp Emerg Care 2010;14:78-84.

8. Reynolds JC, Salcido DD, Menegazzi JJ. Conceptual models of coronary perfusion pressure and their relationship to defibrillation success in a porcine model of prolonged out-of-hospital cardiac arrest. Resuscitation 2012;83:900-6.
9. Metzger AK, Herman M, McKnite S, Tang W, Yannopoulos D. Improved cerebral perfusion pressures and 24-hr neurological survival in a porcine model of cardiac arrest with active compression-decompression cardiopulmonary resuscitation and augmentation of negative intrathoracic pressure. Crit Care Med 2012;40:1851-6.

10. Berg RA, Otto CW, Kern KB, et al. High-dose epinephrine results in greater early mortality after resuscitation from prolonged cardiac arrest in pigs: a prospective, randomized study. Crit Care Med 1994;22:282-90.

11. Ryu HH, Moore JC, Yannopoulos D, et al. The effect of head up cardiopulmonary resuscitation on cerebral and systemic hemodynamics. Resuscitation 2016;102:29-34.

12. Yang $Z$, Tang D, Wu $X$, et al. A tourniquet assisted cardiopulmonary resuscitation augments myocardial perfusion in a porcine model of cardiac arrest. Resuscitation 2015;86:49-53. 